\title{
ON UNIT-ZERO BOOLEAN REPRESENTATIONS OF OPERATIONS AND RELATIONS*
}

BY B. A. BERNSTEIN

1. Introduction. Consider an algebra $(K,+, \mathrm{X})$, such as ordinary real algebra, in which there are two elements " 0 " and " 1 " having the properties that, for any element $a$,

$$
a+0=0+a=a, a 1=1 a=a .
$$

Let

$$
\left(x_{1}, x_{2}, \cdots, x_{m} ; a_{1}, a_{2}, \cdots, a_{m}\right)
$$

denote a unit-zero function with respect to the sequence of $m$ elements, $a_{1}, \cdots, a_{m}$ of $K$, that is, a function $f\left(x_{1}, x_{2}, \cdots, x_{m}\right)$ of $m$ elements $x_{1}, x_{2}, \cdots, x_{m}$ such that $f=1$ or 0 , according as the equalities, $x_{i}=a_{i},(i=1,2, \cdots, m)$, all hold or do not all hold. Accordingly, $(x ; a)$ will denote a unit-zero function with respect to $a$, that is, a function $f(x)$ such that $f(x)=1$ or 0 , according as $x=a$ or $x \neq a$. Then the following propositions (2)-(4) evidently hold:

(2) $\left(x_{1}, x_{2}, \cdots, x_{m} ; a_{1}, a_{2}, \cdots, a_{m}\right)=\left(x_{1} ; a_{1}\right)\left(x_{2} ; a_{2}\right) \cdots\left(x_{m} ; a_{m}\right)$;

$$
a\left(x_{1}, x_{2}, \cdots, x_{m} ; a_{1}, a_{2}, \cdots, a_{m}\right)=a \text { or } 0,
$$

according as $x_{i}=a_{i},(i=1,2, \cdots, m)$, all hold or do not all hold;

(4) $a\left(x_{1}, x_{2}, \cdots, x_{m} ; a_{1}, a_{2}, \cdots, a_{m}\right)$

$$
+b\left(x_{1}, x_{2}, \cdots, x_{m} ; b_{1}, b_{2}, \cdots, b_{m}\right)=a \text {, or } b \text {, or } 0 \text {, }
$$

according as $x_{i}=a_{i}$ all hold, or $x_{i}=b_{i}$ all hold, or neither $x_{i}=a_{i}$ all hold nor $x_{i}=b_{i}$ all hold, $\left(i=1,2, \cdots, m ; a_{i} \neq b_{i}\right.$ for some $\left.i\right)$. In a previous paper $\dagger$ propositions (1)-(4) were made the basis of a method of obtaining arithmetic representations of arbitrary operations and relations in a finite class of elements. Since

* Presented to the Society, April 11, 1931.

$\dagger$ B. A. Bernstein and N. Debely, A practical method for the modular representation of finite operations and relations, this Bulletin, vol. 38 (1932), pp. 110-114. 
propositions (1)-(4) also hold when the symbols belong to Boolean algebra, the question naturally arises: To what extent can unit-zero functions be used analogously to obtain Boolean representations of arbitrary operations and relations? The object of the present paper is to answer this question.

2. Determination of Boolean Unit-Zero Algebras. The possibility of representing arbitrary operations and relations by unitzero functions of an algebra hinges on the existence in this algebra of a unit-zero function for every sequence of $m$ of its elements. Let us call an algebra which has a unit-zero function for every sequence of $m$ of its elements a unit-zero algebra. I proceed first to determine all Boolean unit-zero algebras.

This determination is made easy by noting at the outset that a unit-zero Boolean function must satisfy proposition (2) above and also that it must be single-valued. We therefore need to look only for Boolean unit-zero functions $f(x)$ of a single variable $x$ of the form*

$$
(x ; a)=(1 ; a) x+(0 ; a) x^{\prime} .
$$

From (5) we see, by putting $a=0,1$, that in a Boolean algebra of two elements, $x$ is the unit-zero function of $x$ with respect to 1 , and $x^{\prime}$ is the unit-zero function of $x$ with respect to 0 ; in symbols,

$$
(x ; 1)=x,(x ; 0)=x^{\prime} .
$$

We have, then, that a two-element Boolean algebra is a unit-zero algebra, the unit-zero functions of one variable $x$ being given by (6).

By (2) and (6), all the unit-zero functions of a two-element Boolean algebra can be readily written down. Thus, the unitzero functions of two variables $x, y$ are given by

$$
\begin{aligned}
& (x, y ; 1,1)=x y, \quad(x, y ; 1,0)=x y^{\prime}, \\
& (x, y ; 0,1)=x^{\prime} y,(x, y ; 0,0)=x^{\prime} y^{\prime} .
\end{aligned}
$$

In general, the unit-zero functions of $m$ variables are the $2^{m}$ constituents in the normal development of 1 with respect to the $m$ variables.

* The usual Boolean notations are employed: $a+b, a b, a^{\prime}, 0,1$ are respectively the sum of $a$ and $b$, the product of $a$ and $b$, the negative of $a$, the zero element, the whole. 
Let us now consider a Boolean algebra $A$ of more than two elements. $A$ must have an element $e \neq 0,1$. Suppose, first, that $A$ has a unit-zero function $f(x)$, of form (5), with respect to $e$. Then

$$
f(e)=1, f(0)=0, f(1)=0, \quad(e \neq 0,1) .
$$

But (i) is inconsistent with (5). Hence, our algebra $A$ has no unit-zero function with respect to a sequence containing the element $e$.

Suppose, next, that the algebra $A$ has a unit-zero function $f(x)$, of form (5) with respect to 0 . Then

$$
f(0)=1, f(1)=0, f(e)=0, \quad(e \neq 0,1) .
$$

Hence, by (5),

$$
f(x)=x^{\prime}, f(e)=0, \quad(e \neq 0,1) .
$$

But equations (iii) are inconsistent. Hence, our algebra $A$ has no unit-zero function with respect to a sequence containing the element 0 .

Similarly, our algebra $A$ has no unit-zero function with respect to a sequence containing the element 1 . Hence, a Boolean algebra of more than two elements has no unit-zero functions at all.

Our main result is, then, the following theorem.

Theorem A. The only Boolean unit-zero algebra is a two-element Boolean algebra.

3. Dual Considerations. By the Principle of Duality in Boolean algebras each of the foregoing propositions about unitzero Boolean functions has a dual proposition corresponding to it. To state these duals, let me use the notion of zero-unit function (to be distinguished from unit-zero function). By a zerounit function of $x_{1}, x_{2}, \cdots, x_{m}$ with respect to the sequence $a_{1}, a_{2}, \cdots, a_{m}$, symbolized by

$$
\left[x_{1}, x_{2} ; \cdots, x_{m} ; a_{1}, a_{2}, \cdots, a_{m}\right],
$$

let us mean a function $f\left(x_{1}, x_{2}, \cdots, x_{m}\right)$ such that $f=0$ or 1 , according as $x_{i}=a_{i},(i=1,2, \cdots, m)$, all hold or do not all hold. The duals of (2), (3), and (4) are, then, respectively $\left(2^{\prime}\right),\left(3^{\prime}\right)$, and $\left(4^{\prime}\right)$ following: 


$$
\begin{gathered}
{\left[x_{1}, x_{2}, \cdots, x_{m} ; a_{1}, a_{2}, \cdots, a_{m}\right]} \\
\quad=\left[x_{1} ; a_{1}\right]+\left[x_{2} ; a_{2}\right]+\cdots+\left[x_{m} ; a_{m}\right] \\
a+\left[x_{1}, x_{2}, \cdots, x_{m} ; a_{1}, a_{2}, \cdots, a_{m}\right]=a \text { or } 1
\end{gathered}
$$

according as $x_{i}=a_{i},(i=1,2, \cdots, m)$, all hold or do not all hold;

$\left(4^{\prime}\right)\left\{a+\left[x_{1}, x_{2}, \cdots, x_{m} ; a_{1}, a_{2}, \cdots, a_{m}\right]\right\}$

$$
\cdot\left\{b+\left[x_{1}, x_{2}, \cdots, x_{m} ; b_{1}, b_{2}, \cdots, b_{m}\right]\right\}=a \text {, or } b \text {, or } 1,
$$

according as $x_{i}=a_{i}$ all hold, or $x_{i}=b_{i}$ all hold, or neither $x_{i}=a_{i}$ all hold nor $x_{i}=b_{i}$ all hold, $\left(i=1,2, \cdots, m ; a_{i} \neq b_{i}\right.$ for some $\left.i\right)$.

The dual of Theorem $\mathrm{A}$ is

TheOREM $\mathrm{A}^{\prime}$. The only zero-unit Boolean algebra is a two-element Boolean algebra.

For a two-element Boolean algebra we have, further:

$$
\begin{aligned}
{[x ; 0] } & =x, \quad[x ; 1]=x^{\prime} ; \\
{[x, y ; 0,0] } & =x+y,[x, y ; 0,1]=x+y^{\prime}, \\
{[x, y ; 1,0] } & =x^{\prime}+y,[x, y ; 1,1]=x^{\prime}+y^{\prime} .
\end{aligned}
$$

In general, the zero-unit functions of $m$ variables are the $2^{m}$ factor-constituents in the dual normal development of 0 with respect to the $m$ variables.

Propositions $\left(2^{\prime}\right)-\left(7^{\prime}\right)$ will be used below in the representation of operations that do not satisfy the condition of closure.

4. Representations. It is now clear to what extent we can apply unit-zero Boolean functions in the representation of arbitrary operations and relations. From Theorem A, we have

Theorem B. A unit-zero Boolean representation of arbitrary operations and relations is possible when and only when the class consists of two elements.

For a two-element class $K$, the theory of Boolean representation follows from propositions (2)-(7) and their duals. If we denote the two $K$-elements by the Boolean symbols 0,1 , the representations of all operations $O$ and relations $R$ in $K$ are covered by the cases 1-3 following.

CASE 1. $O$ an m-ary operation satisfying the condition of closure. There is a $K$-element, 0 or 1 , for every sequence $e_{1}, e_{2}, \cdots, e_{m}$ taken from $K$. Let the sequences to which 1 corresponds be 
(i) $\alpha_{11}, \alpha_{12}, \cdots, \alpha_{1 m} ; \alpha_{21}, \alpha_{22}, \cdots ; \alpha_{2 m} ; \cdots ; \alpha_{k 1}, \alpha_{k 2}, \cdots, \alpha_{k m}$.

The representation of $O$ is the Boolean function

$$
\sum_{i=1}^{k}\left(x_{1}, x_{2}, \cdots, x_{m} ; \alpha_{i 1}, \alpha_{i 2}, \cdots, \alpha_{i m}\right) .
$$

CASE 2. O an m-ary operation not satisfying the closure condition. There are sequences in $K$ to which no $K$-elements correspond. Let these sequences be

(ii) $\beta_{11}, \beta_{12}, \cdots, \beta_{1 m} ; \beta_{21}, \beta_{22}, \cdots, \beta_{2 m} ; \cdots ; \beta_{k 1}, \beta_{k 2}, \cdots, \beta_{k m}$.

Consider the operation $O^{\prime}$ obtained from $O$ by assigning a $K$ element, 0 for convenience, to each of the sequences (ii). Let $\phi\left(x_{1}, x_{2}, \cdots, x_{m}\right)$, obtained as in Case 1 , be the representation of $O^{\prime}$. Then the representation of $O$ is the function

(9) $\phi\left(x_{1}, x_{2}, \cdots, x_{m}\right)+\sum_{i=1}^{k} 0 /\left[x_{1}, x_{2}, \cdots, x_{m} ; \beta_{i 1}, \beta_{i 2}, \cdots, \beta_{i m}\right]$,

where $a / b$ means the unique $K$-element $q$ satisfying the condition $b q=a .^{*}$

CASE 3. $R$ an m-adic relation. Let the sequences which do not satisfy $R$ be

(iii) $\gamma_{11}, \gamma_{12}, \cdots, \gamma_{1 m} ; \gamma_{21}, \gamma_{22}, \cdots, \gamma_{2 m} ; \cdots ; \gamma_{k 1}, \gamma_{k 2}, \cdots, \gamma_{k m}$.

Then the representation of $R$ is the Boolean equation

$$
\sum_{i=1}^{k}\left(x_{1}, x_{2}, \cdots, x_{m} ; \gamma_{i 1}, \gamma_{i 2}, \cdots, \gamma_{i m}\right)=0 . \dagger
$$

Of course, by the Duality Principle, the theory of representation can be stated primarily in terms of zero-unit functions instead of unit-zero functions.

5. Illustrations. The following illustrations, one for each of the above three cases, will make the theory of representation quite clear.

$\alpha$. Let $O$ be the operation defined by

* For a two-element Boolean algebra the quotient can be defined precisely as in ordinary algebra.

$\dagger$ Instead of 0 , we can use 1 in (10), provided (i) are the sequences which do satisfy $R$. 


\begin{tabular}{l|ll} 
& 0 & 1 \\
\hline 0 & 1 & 0 \\
1 & 0 & 1
\end{tabular}.

Its representation is

(ii)

$$
(x, y ; 0,0)+(x, y ; 1,1) \equiv x^{\prime} y^{\prime}+x y .
$$

$\beta$. Let $O$ be the operation

\begin{tabular}{c|cc} 
& 0 & 1 \\
\hline 0 & 0 & 1 \\
1 & - & -
\end{tabular}

where the blanks indicate that there are no $K$-elements corresponding to the sequences 1,$0 ; 1,1$.

Consider the operation $O^{\prime}$ defined by

$$
\begin{array}{l|ll}
- & 0 & 1 \\
\hline 0 & 0 & 1 \\
1 & 0 & 0
\end{array} .
$$

By Case 1, the representation of $O^{\prime}$ is

(v)

$$
x^{\prime} y \text {. }
$$

Hence, the representation of $O$ is

(vi) $x^{\prime} y+0 /[x, y ; 1,0]+0 /[x, y ; 1,1]$

$$
\equiv x^{\prime} y+0 /\left(x^{\prime}+y\right)+0 /\left(x^{\prime}+y^{\prime}\right) \text {. }
$$

$\gamma$. Let $R$ be a relation defined by

$$
\begin{array}{c|cc} 
& 0 & 1 \\
\hline 0 & - & + \\
1 & + & -
\end{array}
$$

where "+" indicates that $R$ holds and "-" indicates that $R$ does not hold. Its representation is the equation

(viii) $\quad(x, y ; 0,0)+(x, y ; 1,1) \equiv x^{\prime} y^{\prime}+x y=0 .^{*}$

The University of California

* For a complete set of Boolean representations of binary operations and dyadic relations in a two-element class, obtained from considerations other than the above, see my Complete sets of representations of two-element algebras, this Bulletin, vol. 30 (1924), pp. 24-30. 